\title{
Describing Ontology Applications
}

\author{
Thomas Albertsen and Eva Blomqvist \\ Jönköping University, Jönköping, Sweden \\ $\{$ alth|blev\}@ing.hj.se
}

\begin{abstract}
Semantic Web technologies are finally, after a few years of infancy, truly entering the business world to support the growing needs of computer aided information selection and processing. There are already quite well-defined development processes and methods in the software engineering field to handle the construction of large scale and complex enterprise systems, and to reuse knowledge in different software domains patterns are considered to be common practise. Patterns can be described on different levels of abstraction, but the patterns in the focus of this paper are on the software architecture level. In this paper we present a definition of the notion "ontology application pattern", as a special form of software architecture patterns describing an ontology-based system. We also show how such patterns, as well as the description of the pattern instantiations, can be described using a modified architecture description language.
\end{abstract}

\section{Introduction}

In recent years the area of semantic applications has grown from small web applications, to large scale enterprise systems showing real benefits in the business applications domain. Technologies intended for the web are now also applied on company intranets and in enterprise information systems etc. As the technology development increases system capabilities, the systems keep getting larger and more complex. Semantic applications are comparable to any other software systems in terms of size and complexity, and thereby require the same rigorous development process as any other system.

Complexity of a system to be built can be managed through established development processes and knowledge reuse (through for example patterns), this is common practise in software development. When constructing semantic applications ontologies are usually used as the core knowledge component of the system. This paper addresses the issue of specifically incorporating ontologies in the architecture design of a software system through a specialisation of software architecture patterns, and extending an architecture description language to better suit software architectures including ontologies.

The following section presents motivation and background of our approach, including related work on patterns and architecture description languages. In section 3 the term ontology application pattern is defined and described in more 
detail. A way of describing the specific kinds of software architectures are discussed in section 4 . Finally, the paper is concluded with a discussion and some future work possibilities.

\section{Background}

This section describes the general background, including definitions, and some previous work on patterns.

\subsection{Ontologies and Patterns}

Ontology is a popular term today, used in many areas and defined in many different ways. In our research we adopt the definition from 11, stating that an ontology is a formal explicit specification of a shared conceptualisation. In our view this means that an ontology is a hierarchically structured set of concepts describing a specific domain of knowledge, that can be used to create a knowledge base. An ontology contains concepts, a subsumption hierarchy, arbitrary relations between concepts, and possibly other axioms.

Even using this definition, ontologies can be used for many different purposes and applications, and they can be constructed and structured in many different ways. Our research focuses mainly on the abstraction level of domain and application ontologies (as defined in 2]) within enterprises, so called enterprise application ontologies, to be used in enterprise applications. When considering the complexity of the ontologies, our research is mainly focused on terminological ontologies, to structure and retrieve information.

In a previous paper [3] we have attempted to describe and classify different kinds of patterns concerning ontologies. In general, patterns are here used in the sense of a generalised solution to a recurring problem that can be reused (instantiated and adapted) in the specific engineering task at hand. In 3. the patterns concerning ontology engineering are divided into five intuitive levels of abstraction. The four lowest levels deal with the internal structure of the ontologies, so called ontology patterns, while the fifth level deals with complete ontologies used within a system, thereby making the connection to software engineering and software architectures. This level of abstraction is in [3] denoted "ontology application patterns" and will be the focus of the rest of this paper.

\subsection{Ontology Applications}

There are a lot of applications that use ontologies today. In 4 a description of four classical ontology application scenarios is made: Neutral authoring, common access to information, ontology as specification and semantic search. Another description of ontology-based applications, more focused on the business domain, is the classification in [5]. Four slightly different scenarios are described: Knowledge management, information retrieval, portals and web communities and finally e-commerce. 
The trend is now to move away from traditional heavily knowledge based systems, using a single ontology, to more open semantic applications (as for example stated in [6]) exploiting and handling the huge size of the web or company intranets and the diversity of both ontologies and information sources. As stated already in the introduction of this paper, these kinds of semantic applications are entering also the business area, through for example intranets, company portals and e-commerce. As technologies develop, more advanced applications become possible, which also increases the complexity and size of the software systems that use the ontologies.

To describe a whole ontology and its use in an application there is a need to describe the characteristics of the ontology. A formulation of such characteristics has been described in connection with the Ontology Definition Metamodel presented by the Object Management Group (OMG) in [7, the dimensions described there are:

- Level of Authoritativeness: This is a measure of how authoritative the ontology is of the area it describes. If the author of the ontology is the organisation that is responsible for specifying the conceptualisation then this is clearly a highly authoritative ontology.

- Source of Structure: Either the ontology is developed separately from the application that will use it or else the structure comes directly from the intended application.

- Degree of Formality: Degree of formality refers to the level of formality of the specification of the conceptualisation. This could range from highly informal ontologies, via semantic networks, to highly formal ontologies that include complex logical axioms.

- Model Dynamics: This concerns the rate of change in the ontology, from the extreme where the ontology is stable and rarely or ever changes, to very volatile ontologies that change continuously.

- Instance Dynamics: This dimension is closely related to Model Dynamics but concerns the instances of the ontology.

- Control and Degree of Manageability: This dimension considers who decides when and how to change an ontology. One extreme is that the author of the ontology has the sole decision on changes, and the other extreme is of course that the ontology changes are based on decisions of outside parties.

- Application Changeability: The applications that use the ontology might be on one hand developed once and for all, and not frequently changed, and on the other hand they might be changing dynamically during run time.

- Coupling: This dimension describes how closely coupled applications committed to shared ontologies are.

To further describe the ontology and what content it should have, competency questions could be used. Competency questions, as described in [8], are used to exactly define what information the ontology should provide, in terms of constraints on its content. A competency question can be expressed as a constraint, but it also contains information on the content of the ontology. Competency 
questions can be expressed in formal logic or more informally, as requirements on the ontology. In this paper we mainly consider informally expressed competency questions.

Other requirements that should be specified according to most common ontology development methodologies, as described in [9], concern:

- Purpose and Goal: The intended use of the ontology.

- Scope: Describing major subject areas that need to be covered in the ontology, and possible boundaries to what is not to be included.

- Level of Detail: Specifying the lowest level of detail considered important (the instance level).

- Representation Suggestions: Suggestions of naming conventions, representation language appropriateness etc.

Often a system could exploit not only one ontology but several, perhaps even exchangeable during runtime. Then these ontologies might need a connection, in order to for example translate information expressed in one ontology to the other. This process of translation requires an ontology alignment to be present, which can be obtained through an ontology matching process as defined in [10].

\subsection{Software Architectures and Patterns}

A description of a software architecture is the description of the high level structure of a software system. In [11] the term software architecture is defined as "the description of elements from which systems are built, interactions among those elements, patterns that guide their composition and constraints on these patterns. [...] a particular system is defined in terms of a collection of components and interactions among those components".

This definition describes the overall design of the system that includes global control structure, communication protocols, data access, and the system's major components and the behaviour of the components. The essential idea of architecture is abstraction, to hide some of the details of the system in order to make it easier to understand the properties of the system, and to connect the functionality in the requirements to elements of the high level design. If the system is complex there can be several levels of abstraction, and the elements on each level can be decomposed into new architectures.

The elements of an architecture can as noted above be divided into components, connectors and their configuration. Both components and connectors provide interfaces that act as connection points to that entity. Sometimes also the data that is exchanged between components is included in the architectural view (see for example [12]). The component is classically an abstract unit of software instructions and internal state that provides a transformation of data. This can be transformations as computation or as simply loading data to memory from secondary storage. The connectors are an abstract mechanism that facilitates communication between the components. A connector may have a subsystem inside it, in order to make this communication possible. Data is the information that is transferred between the components through the connectors of the architecture. 
An architecture style describes a generalised architectural organisation of software systems, which means that it describes how components and connectors can be configured into a certain kind of software system with certain properties. In [11 the notion of architecture style is defined as follows: ".. an architectural style defines a vocabulary of components and connector types, and a set of constraints on how they can be combined." It is also stated that many styles might in addition include a semantic model that defines how to determine the system's overall properties from its parts.

Examples of common architecture styles are "Pipes and Filters" where the components are filters while the connectors are the pipes. Another architecture style is the "Object Oriented" style. The components encapsulate the data and the operations, as connectors, make it possible for the components, the objects, to communicate. The connectors are usually procedure calls. If an architecture style is more formally described it constitutes and architecture pattern, such as the patterns in [13] and [14].

\subsection{Architecture Description Languages}

In order to support the description and communication of software architectures the use of architecture description languages (ADLs) have been proposed. An ADL is usually described as a language designed for describing high level architectural notions, including components, connectors, and their interfaces, and that treats connectors as first-class entities. ADLs were originally developed when existing formal languages and programming languages were found to be insufficient or inappropriate to describe a software architecture (see discussion in [1].).

For software ADLs the components in the language are software processes or modules and the ADL is used to define and model the software architecture. There are numerous ADLs existing today, examples are Rapide, UniCon, Darwin and xADL. In order to transfer an architecture description from one ADL to another the ADL ACME has been constructed to facilitate mapping from one ADL to another. For a survey and comparison of ADLs see [15].

xADL (see [16]) has been developed by the University of California Irvine and is defined as a set of XML schemas. The language has a core model with four elements: Components, connectors, interfaces and configurations. These are mainly the same elements as discussed in section 2.3. The language has a modular design that makes it easy to extend with new structures.

\section{Ontology Application Patterns}

In this section we attempt to define the notion of ontology application patterns, as an extension of software architecture patterns. As software architecture patterns are usually described using a special template, one such template is described and detailed to better fit ontology applications. 


\subsection{Definition}

Ontology application patterns are very much related to software architecture patterns, although ontology application patterns include ontologies as components. Our definition of ontology application patterns therefore build on the definition of software architecture patterns stated in section 2.3 .

We define an ontology application pattern as "a software architecture pattern describing a software system that utilises ontologies to create some of its functionality. The pattern also describes properties of the ontology or ontologies in the system, and the connection between the ontology, other ontologies and the rest of the system".

\subsection{Specific Characteristics}

The difference between software architecture patterns and ontology application patterns is that there always exist ontology components in the latter and that the pattern should give insight in what capabilities these ontology components should have. First we consider the topology of the complete architecture, to address the nature of ontology components. How are the ontology components used in the complete system? There can be several ways to view this, on several levels of abstraction. If the abstraction of the system is high, the ontology component might include a lot of "intelligence" such as an inference engine and additional software functionality. If on the other hand the abstraction level is low, the component might represent only the ontology, as a data source. These two views imply different interfaces and connectors of the ontology component, but both views might be used for describing patterns as well as for describing a specific software architecture.

To give a simple example, we use a scenario from a research project at Jönköping University called MediaILOG. The project aims at introducing semantic technologies to address information processing problems within companies of the media industry. Specifically a local newspaper of the Jönköping region is used as an application case of the project. One part of a proposed framework in the context of this project is the possibility to rank incoming documents (whether they are e-mails, articles from a news agency or update reports from a website) with respect to the company's interests (as represented in their enterprise ontology) and the individual interests or job descriptions of the employees of the company (also described in the enterprise ontology). This process should be supported by a software system for evaluating and ranking incoming documents. A simplified version of such an architecture can be depicted as in figure 1 where part A illustrates the architecture on a high level of abstraction, the enterprise ontology is an internal part of the profile matching component, and part B illustrates the case when the ontology is viewed as a component in itself.

In both views described above the internal structure and content of the ontology itself needs to be considered. This leads to the question, what characteristics should be described concerning an ontology in an architecture or even an ontology application pattern? In section 2.2 a set of ontology characteristics are given, along with the requirements usually used when constructing ontologies 
A)

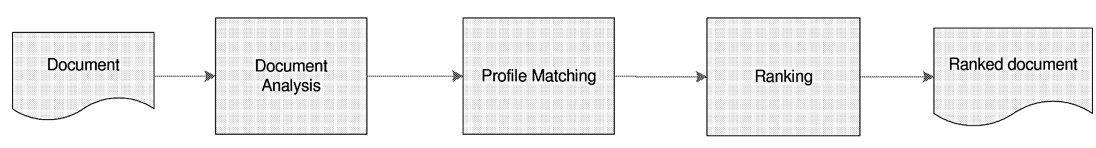

B)

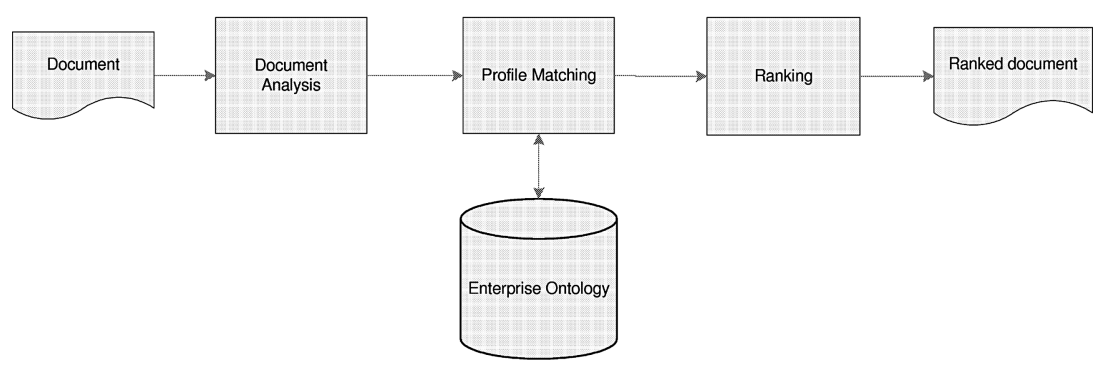

Fig. 1. Examples of architecture alternatives

(like scope, level of detail and a set of competency questions). Together this can be viewed as a description characterising the ontology as well as requirements for its construction. In the example architecture such information would include the scope of the enterprise ontology, the required level of detail and a set of competency questions describing the ontology capabilities.

There can be competency questions on several levels; general abstract questions stated for a pattern, and more specific ones developed when implementing the pattern. An ontology pattern used for ontology construction might describe the content and internal structure of a part of the ontology in general terms, like the ontology design patterns of [17, and could be used as a way to describe the content requirements of an ontology. If for example the ontology application pattern would suggest the use of a structure similar to the DOLCE roles and tasks pattern in 17] (describing how objects and events in a certain situation are described by roles and tasks) it could also include suggestions for competency questions, representing an abstract interface to the ontology. An example is to be able to "ask" the ontology "what role has a certain object that participates in this specific event". When the pattern is instantiated such a question can be specified into "what role has this person participating in this meeting". An ontology application pattern might suggest ontology patterns for realising the ontology component and competency questions for realising its interface.

In the example of figure 1 such description of the ontology interface (of alternative B) could include competency questions like i) "what are the related topics of a topic $t$ " or ii) "what are the interests of employee $e$ ". This should be part of the component description of an ontology component in a software architecture and thereby also, in more general terms, might be addressed in 
an ontology application pattern. Such a more general version of the competency question ii could for example be "what are the concepts considered relevant from the perspective of instance $i$ ". In addition, if the more general view (alternative A) is adopted, including software structures in the ontology component, also the functionality of the surrounding software and its interface should be described.

Another issue when considering interfaces to ontology components are the possible and intended connections. The above discussed example component interfaces support mainly the connection of an ontology component to a pure software component. Another possibility is to connect an ontology component directly to another ontology component. Then the interface might constitute either only the competency questions (for dynamic connections) or the complete internal structure of the ontology (for more static approaches). Interfaces can be described both as general types, for ontology application patterns, and as specific instances of those types for the instantiation of the patterns.

When connecting two ontology components directly the connector between these probably contains an ontology alignment (see section 2.2). The interface of the ontology components are in the case of a static alignment the complete ontology, since the alignment can operate on any internal part of the ontologies. In the case of a dynamically constructed alignment the interface is a set of competency questions and the connector a set of queries to construct the alignment. On a pattern level this can be described as an abstract alignment, an alignment between components, but on the level of the specific pattern instantiation the nature of the alignment might be further specified.

An example of this situation could also be illustrated through a simplified part of the framework envisioned in the MediaILOG project previously mentioned. When collecting relevant information from news agency services the enterprise ontology of the newspaper can be aligned to ontologies describing the structure and general content of the information provided by different news agencies, as illustrated in figure 2 In this way when the newspaper wants to retrieve information from a news agency a wrapper interpreting the alignment between the enterprise ontology and the news agency ontology is used, to translate the information need of the newspaper into a query to be sent to an appropriate news agency. In this simplified example, there exists a static connection between the enterprise ontology and several news agency ontologies. Each ontology acts as a component of this architecture and the alignments act as the connector component between ontology components. But in practise it may also be used by the wrapper components, as illustrated in figure 2, which gives the wrappers access to the aligned ontologies.

\subsection{Pattern Template}

In order to make it easier to create ontology application patterns a template, corresponding to the commonly used template in [18] is detailed to better fit ontology application patterns. The suggested template in [18] has the following headings:

- Name of pattern

- Aliases: Aliases, if there are any. 


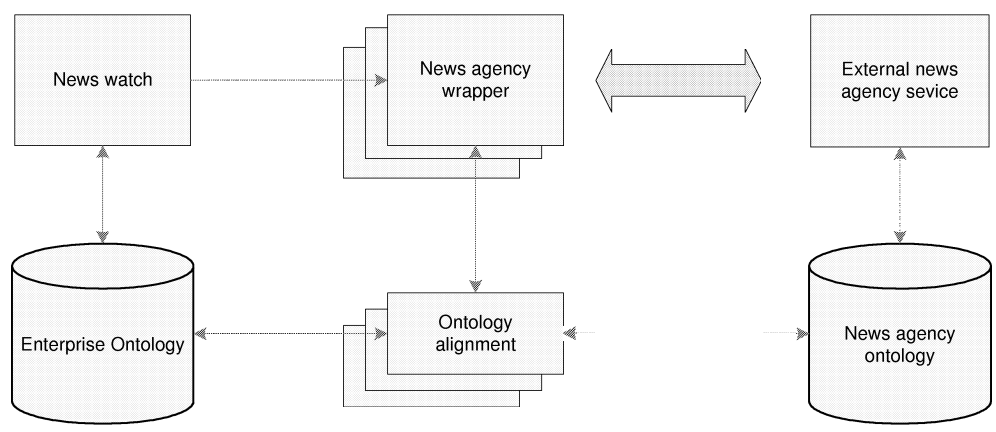

Fig. 2. Example of an architecture involving static alignments

- Problem: Gives a short description of the problem. This could be a statement or a question.

- Context: The context in which the pattern is valid.

- Forces: Lists and describes each relevant force. A force is a factor or attribute relevant to the problem, the solution is the result of the "tension" between the forces. Examples of forces can be factors such as efficiency or robustness. Forces can be conflicting and the solution should balance the tension between the forces present in the problem.

- Solution: Gives a statement of the solution to the problem, can include diagrams.

- Resulting Context: Describes the context after the solution is applied.

- Rationale: Explains the logic behind the solution. The user will understand why this solution is a good solution.

- Known Uses: Gives examples on where the pattern have been used.

- Related Patterns: Lists any related patterns.

- Sketch: A sketch to describe the pattern and a description of the sketch, if needed.

- Author(s): Names of the authors.

- Date: Date created.

- Contact Details: Authors' emails

- Pattern Source: The source of the pattern, for example the affiliation of the authors.

- References: A list of references cited in the pattern.

- Keywords: A string of comma delimited terms used for searching.

- Example: Referenced in the pattern. This could be pseudo code, skeleton for classes etc.

To further detail this template for ontology application patterns, information about the ontology components need to be specified in the template sections. In the following list subheadings illustrate where new information should be added:

- Context

- Application Context 
- Knowledge Context: Describing the context, especially regarding level of authoritativeness, model and instance dynamics, application changeability and coupling, for the pattern to be applicable.

- Forces

- General Forces

- Ontology Forces: Describing forces related to the use of ontologies and the requirements set by the pattern on the ontology.

- Solution

- Software Solution

- Ontology Solution: Describing the intention and general requirements set on the ontology by the pattern, like purpose, scope, source of structure, level of detail, degree of formality, representation suggestions (and reasoning capabilities) and a set of general competency questions.

- Resulting Context

- Application Context

- Knowledge Context: Describing the model and instance dynamics, application changeability and control, and degree of manageability.

- Example: The examples might include parts of ontologies and references to reusable ontologies.

\section{Architecture Description}

When using the above described template much of the information regarding an ontology application pattern can be captured, but only in the ambiguous manner of arbitrary textual descriptions, simple illustrations and examples. To more formally describe a pattern, or a pattern instance implementation, a structured language (such as an ADL) is needed. Existing ADLs focus on software components and the interactions between them. We feel there is a need for focusing more on knowledge components to be able to define an ontology application pattern or an application architecture containing ontologies properly. Many of the existing ADLs are possible to use for describing ontology components, but to capture the above noted characteristics of ontology components and connectors the languages have to be extended.

\subsection{Suggested ADL}

Architecture description languages usually contain the elements components and connectors. If ontologies are treated as another component there are several issues to be considered, as described in section 3. If the component description in an ADL were to be extended by the characteristics of the ontology this could be a way to show what the ontologies must be able to do within the component and the complete system.

As described in section 2.4 there are several ADLs available. In order extend an ADL to accommodate the use of ontologies an ADL with the property of being easily extendible were chosen, namely xADL 2.0. xADL has a modular 
organisation, where new modules can be added to include more specific expressions in the language. This is a good way to create a description of a system using ontologies i.e. creating an ADL for ontology applications.

Ontologies can be seen as components in the system with some sort of information inside and given interfaces. Other components within the system should be able to access and manipulate the ontology. With this in mind, a component with the additional properties of an ontology should be added to the language. The characteristics mentioned in 2.2 are used as additional properties of the ontology component and thereby a new element called ontology is created in xADL. The ontology element has a description element called the Ontology Description, which has all the ontology characteristics as attributes. This is illustrated in figure 3 through a graph based notation provided by xADL 2.0.

The interfaces are the connection points to the ontology component and should describe what services the ontology can provide to the other components through some connector. In section 3.2 examples of such services are given, and those are examples of what can be considered ontology interfaces. Mainly there are two kinds of interfaces; interfaces directly to another ontology component and software interfaces (possibly constrained by some competency questions). The interface between ontologies also has two variants, either constituting the complete ontology or just the set of competency questions. These variants are added as specialised interface description attributes as illustrated in figure 4 .

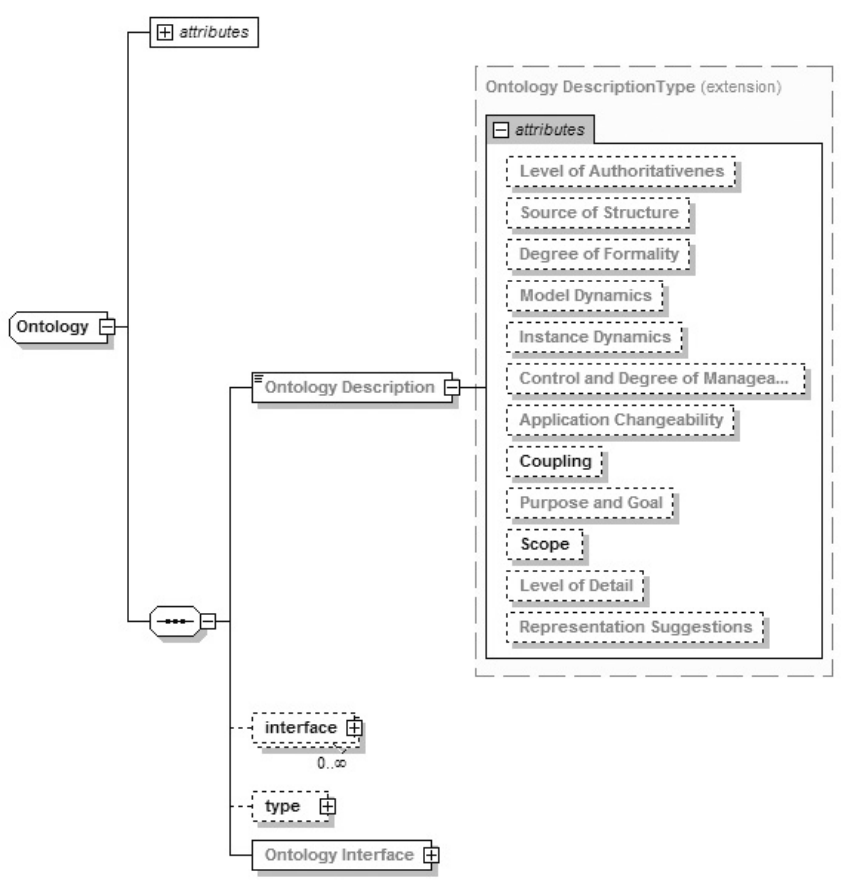

Fig. 3. Ontology component 


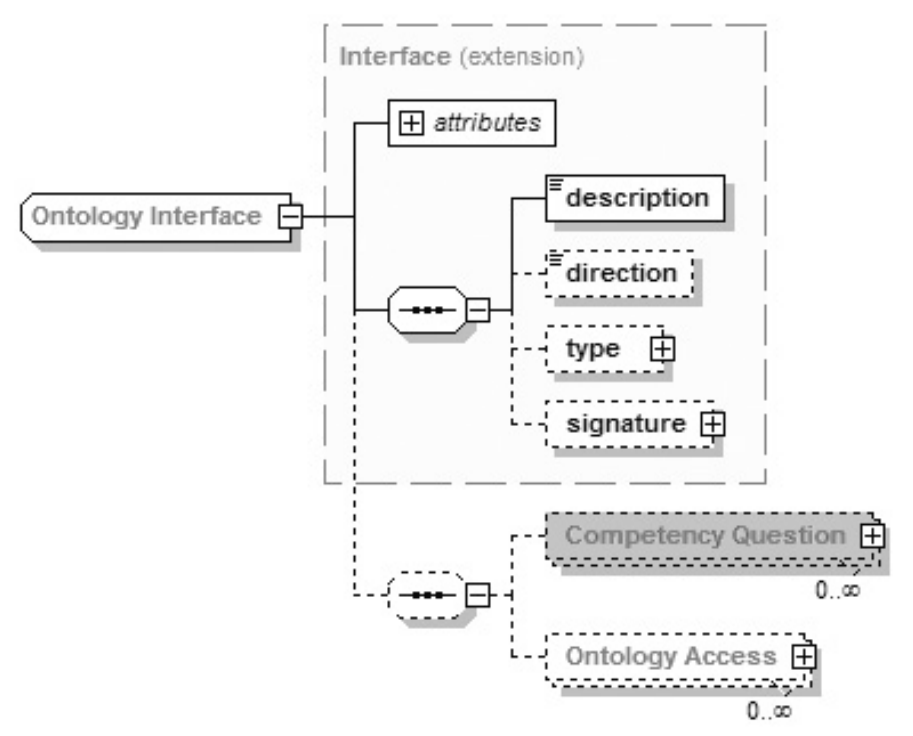

Fig. 4. Ontology interface

The last of the added elements is the connector, that will make communication to and from the ontology components possible. Connectors can also be of several kinds, but if the ontology is connected to a software component there is no major difference to an ordinary software connector, only the possible addition of constraints (competency questions). If the ontology is on the other hand connected to another ontology component directly, the connector might be a set of queries or a set of competency questions, or both. These are added as a specialised attribute in the ontology connector as illustrated in figure 5 .

\subsection{Architecture Example}

When considering the simplified project scenarios discussed earlier in section 3.2 the first architecture (version B of figure 1) could for example be described using the suggested additions to xADL. Due to space limitation we can here only give a brief idea of what this description could contain (using the added attributes of ontology components, connectors and interfaces).

The ontology component is in this example architecture considered as a data store with no software functionality of its own. The description of this enterprise ontology using the component template depicted in figure 3 should include information of all the attributes of an ontology component. Level of authoritativeness would in this case describe the level of authoritativeness required from the ontology to support accurate ranking of incoming documents. The level of authoritativeness should be high in order for the rankings to be useful, so the ontology needs to be tailored to the media company at hand. Source of structure would include information of the sources for ontology construction, in the case 


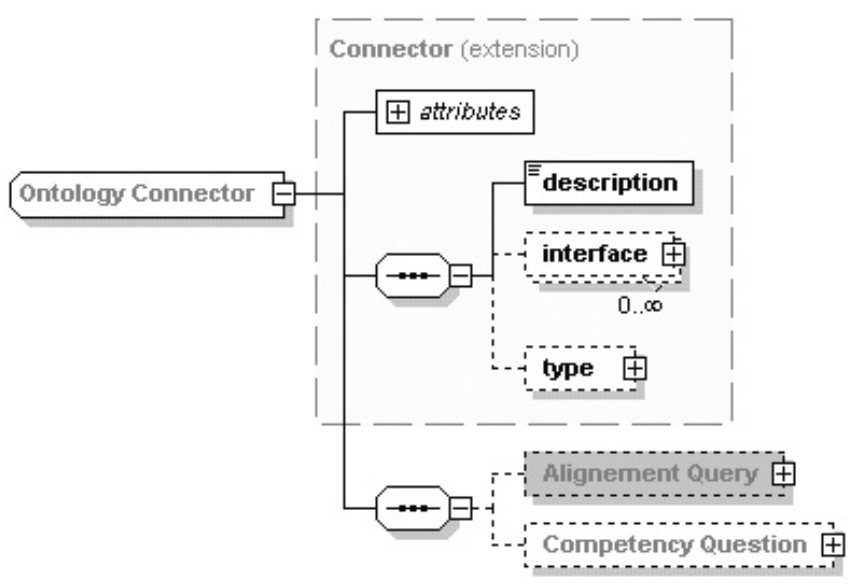

Fig. 5. Ontology connector

of a local newspaper this might be the paper sections and the actual work division structure between departments and individual employees. The structure of the ontology needs to be tailored to the task it should perform. The degree of formality states if informal definitions are acceptable or if each concept needs a formal logical definition. In this application case a semantic net representation of the ontology is sufficient. Model dynamics describes how dynamic the enterprise ontology will be, in this case a traditional newspaper with fixed topics probably has a quite static enterprise ontology. On the other hand, the level of detail needs to be high, which gives a higher level of instance dynamics. This kind of reasoning can be performed for each of the attributes of the ontology component, based on the application case requirements.

As an interface description the competency questions of the ontology should be one part, in addition the representation language of the ontology needs to be specified. For the example case the following two competency questions were suggested in a previous section: "what are the related topics of a topic $t$ " and "what are the interests of employee $e$ ". The ontology representation is needed to support the implementation of the connector interface. Finally, the connector between the ontology component interface and the software component (in the example denoted "profile matching") needs to be specified. This will be the reasoning software used to query the ontology, for example using some ontology query language, allowing queries corresponding to the competency questions of the interface. This reasoner will then connect to the software interface of the profile matching component, thereby letting that component use the services corresponding to the competency of the ontology.

So far we have not generalised these example architectures into patterns, but we envision that several architecture patterns can be created in each application field (as discussed in section 2.2) simply by looking at existing systems. The extended version of xADL presented above can equally well be used to describe 
those patterns. All attributes may not apply for every pattern, but generally the attributes are still valid. The modified pattern template suggested in section 3.3 can be viewed as the natural language correspondence to the added component templates in xADL.

\section{Conclusions and Future Work}

In this paper we have defined the notion of ontology application patterns, as a specialisation of software architecture patterns. Then we have continued to study the characteristics that differentiate these patterns from ordinary software architecture patterns. Mainly we can see that engineering ontology applications may benefit from descriptions and patterns more focused on ontologies and their use in the system. Therefore we propose a more detailed template to guide the future description and documentation of ontology application patterns. A next step will be to construct a catalogue of ontology application patterns according to our definition and defined using the extended template.

Both pattern descriptions and architecture descriptions in themselves can also benefit from being described in a more formal manner. Therefore we have extended the general architecture description language xADL 2.0 with specific ontology component templates, together with specialised connectors and interfaces. Future work will involve to evaluate the extended ADL through applying it in a set of cases and determine the completeness of the attributes. It will also be used to describe future system architectures of ontology applications developed by our research group, in projects like MediaILOG.

\section{Acknowledgements}

Examples used in this paper were provided by the MediaILOG research project at Jönköping University, financed by the Swedish Hamrin Foundation. Also special thanks to the three anonymous reviewers for their valuable comments on how to improve this paper.

\section{References}

1. Gruber, T.: A translation approach to portable ontology specifications. In: Knowledge Acquisition. Volume 5. (1993) 199-220

2. Guarino, N.: Formal Ontology and Information Systems. In: Proceedings of FOIS'98. (1998) 3-15

3. Blomqvist, E., Sandkuhl, K.: Patterns in Ontology Engineering: Classification of Ontology Patterns. In: Proc. of ICEIS2005, Miami Beach (2005)

4. Jasper, R., Uschold, M.: A Framework for Understanding and Classifying Ontology Applications. In: Proceedings of the Twelfth Workshop on Knowledge Acquisition Modeling and Management KAW'99. (1999)

5. OntoWeb: D21 Successful Scenarios for Ontology-based Applications v1.0. OntoWeb Deliverable, D2.1, Available at: http://www.ontoweb.org/About/ Deliverables/ (2002) 
6. Motta, E., Sabou, M.: Next Generation Semantic Web Applications. In: Proceedings of the First Asian Semantic Web Conference - ASWC 2006, Springer (2006)

7. Object Management Group: Ontology definition metamodel. IBM and Sandpiper Software Inc, Available at: http://www.omg.org/docs/ad/06-05-01.pdf (2006)

8. Gruninger, M., Fox, M.S.: The Role of Competency Questions in Enterprise Engineering. In: IFIP WG5.7 Workshop on Benchmarking - Theory and Practice, Trondheim, Norway (1994)

9. Öhgren, A., Sandkuhl, K.: Towards a methodology for ontology development in small and medium-sized enterprises. In: IADIS Conference on Applied Computing, Algarve, Portugal (2005)

10. Shvaiko, P., Euzenat, J.: A Survey of Schema-based Matching Approaches. Journal on Data Semantics (2005)

11. Shaw, M., Garlan, D.: Software Architecture - Perspectives on an Emerging Discipline. Prentice-Hall, Upper Saddle River (1996)

12. Fielding, R.T.: Architectural Styles and the Design of Network-based Software Architectures. PhD thesis, University of California, Irvine, Irvine, California (2000)

13. Buschmann, F., Meunier, R., Rohnert, H., Sommerlad, P., Stal, M.: Patternoriented Software Architecture - A System of Patterns. John Wiley \& Sons (1996)

14. Fowler, M.: Patterns of Enterprise Application Architecture. Addison-Wesley (2003)

15. Medvidovic, N., Taylor, R.: A Classification and Comparison Framework for Software Architecture Description Languages. IEEE Transactions on Software Engineering 26 (2000) 70-93

16. Dashofy, E.M., van der Hoek, A., Taylor, R.N.: A Highly Extensible, XML-Based Architecture Description Language. In: Proc. of WICSA 2001. (2001)

17. Gangemi, A.: Ontology Design Patterns for Semantic Web Content. In: Proceedings of ISWC2005, Springer (2005) 262-276

18. Meszaros, G., Doble, J.: Metapatterns: A pattern language for pattern writing. In: The 3rd Pattern Languages of Programming conference, Monticello, Illinois (1996) 